\title{
EFECTO ESTROGÉNICO DEL EXTRACTO HIDROALCOHÓLICO DE Medicago sativa L (ALFALFA) EN RATAS ALBINAS OVARIECTOMIZADAS
}

\author{
Estrogenic effect of Medicago sativa L (alfalfa) hydroalcoholic extract in albino rats ovariectomized
}

\author{
Sandra G. Bezada ${ }^{1}$, Jorge L. Arroyo², Martín Condorhuamán ${ }^{3}$
}

${ }^{1}$ Facultad de Medicina Veterinaria. ${ }^{2}$ Facultad de Medicina Humana. ${ }^{3}$ Instituto de Investigación en Ciencias Farmacéuticas y Recursos Naturales "Juan de Dios Guevara", Facultad de Farmacia y Bioquímica. Universidad Nacional Mayor de San Marcos

\section{RESUMEN}

El presente estudio tuvo como objetivo demostrar las propiedades estrogénicas del extracto hidroalcohólico de $M e d i c a g o$ sativa L (alfalfa) en ratas albinas ovariectomizadas (OVX). Se utilizaron 48 ratas albinas Sprague Dawley, hembras, de 200 a $250 \mathrm{~g}$, de 8 semanas de edad, de las cuales 40 fueron sometidas a extirpación quirúrgica de ambos ovarios siguiendo la técnica de ovariectomía bilateral por el flanco. Luego del post-operatorio se dividieron en seis grupos y se administró el extracto hidroalcohólico por vía oral bajo el siguiente esquema de trabajo: Grupo 1 control negativo: (OVX), vehículo del

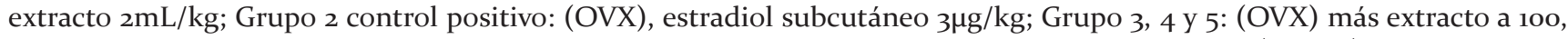
500 y $1000 \mathrm{mg} / \mathrm{kg}$, respectivamente; y Grupo 6: No OVX, control del procedimiento quirúrgico (SHAM). El tratamiento duró 14 días y se evaluó el peso corporal, peso del útero, cambios del ciclo estral mediante frotis vaginal y análisis del perfil hormonal. Se observó aumento en el peso corporal, en el peso del útero, en los niveles del estradíol y cambios en la citología vaginal. Se concluye que el extracto hidroalcohólico de Medicago sativa L (alfalfa) presentó efecto estrogénico al modificar los parámetros evaluados.

Palabras clave: Papaya de altura, composición química, valor nutritivo, valor tecnológico-nutricional.

\section{SUMMARY}

This study aimed to demonstrate the estrogenic properties of the hydroalcoholic extract of Medicago sativa L (alfalfa) in albino rats ovariectomized (OVX). Were used 48 albino Sprague Dawley rats of 200 to 250 g, 8 weeks of age, of whom 40 underwent surgical removal of both ovaries, following the technique of bilateral ovariectomy on the flank. After the postoperative were divided into six groups and hydroalcoholic extract was administered orally under the following scheme of work: Group 1 negative control: (OVX), vehicle of the extract $2 \mathrm{~mL} / \mathrm{kg}$; Group 2 positive control: (OVX), estradiol subcutaneous 3 $\mu \mathrm{g} / \mathrm{kg}$; Group 3, 4 y 5: (OVX) more extract 100, 500 and $1000 \mathrm{mg} / \mathrm{kg}$, respectively; and Group 6: not OVX control the surgical procedure (SHAM). The treatment lasted 14 days and was evaluated the body weight, uterine weight, changes of oestrus by vaginal smears and hormonal profile analysis. Were observed increase in body weight, uterine weight, in estradiol levels and changes in vaginal cytology. Was conclude that the hydroalcoholic extract of Medicago sativa L (alfalfa) showed estrogenic effect by modifying the parameters evaluated.

Keywords: Papaya height, chemical composition, nutritional value, technological and nutritional value.

\section{INTRODUCCIÓN}

a deficiencia de estrógenos, asociada con la menopausia en la mujer, condiciona a la aparición de síntomas denominados "sofocos", predisposición a osteoporosis, enfermedad cerebro-vascular, disminución de la memoria de corto plazo e incluso atrofia y sequedad vaginal, lo que disminuye la calidad de vida en la mujer ${ }^{(1-4)}$. Si bien el tratamiento de primera línea para el cese o disminución de estos síntomas continúa siendo la terapia de reemplazo hormonal, en la actualidad muchas mujeres vienen optando por el uso de remedios botánicos con indicios de actividad estrogénica debido a los riesgos que conlleva el uso prolongado de hormonas sintéticas ${ }^{(5,6)}$.
Es de conocimiento general que la mayoría de plantas leguminosas poseen fitoestrógenos, los cuales varían dependiendo de numerosos factores como son la humedad, temperatura, horas-luz, edad de la planta, incluso si se emplea o no abono y el tipo del mismo ${ }^{(7)}$. La alfalfa (Medicago sativa $\mathrm{L}$ ) es un reconocido miembro de esta familia; dentro de su composición química se encuentran fitoestrógenos tales como las coumarinas y pequeñas cantidades de formononetina y biochanina ${ }^{(8,9)}$.

El fitoestrógeno que se encuentra presente en mayor cantidad es el coumestrol, el cual es de 30 a 100 veces más potente que las isoflavonas ${ }^{(9)}$ y 4 'metoxi coumestrol (forma inactiva) ${ }^{(7)}$. Todas estas sustancias estrogénicas de origen vegetal se encuentran en 
Tabla 1. Distribución de grupos para la determinación del efecto estrogénico.

\begin{tabular}{|c|c|c|c|c|c|}
\hline Grupo & Categoría & Técnica & Tratamiento & $\begin{array}{c}\text { V'́a } \\
\text { administración }\end{array}$ & Dosis \\
\hline G-1 & Control Negativo & OVX & Vehículo & Oral & $2 \mathrm{~mL} / \mathrm{kg}$ \\
\hline G-2 & Control Positivo & ovX & Estradiol & SC & $3 \mu \mathrm{g} / \mathrm{kg}$ \\
\hline G-3 & Experimento & ovx & Extracto alfalfa & Oral & $100 \mathrm{mg} / \mathrm{kg}$ \\
\hline$G-4$ & Experimento & ovx & Extracto alfalfa & Oral & $500 \mathrm{mg} / \mathrm{kg}$ \\
\hline G-5 & Experimento & ovX & Extracto alfalfa & Oral & $1000 \mathrm{mg} / \mathrm{kg}$ \\
\hline G-6 & $\begin{array}{c}\mathrm{CPQ} \text { (Control } \\
\text { procedimiento } \\
\text { quirúrgico) * }\end{array}$ & $\begin{array}{c}\text { SHAM } \\
\text { (No OVX) }\end{array}$ & Alimento/agua & Oral & Ad libitum \\
\hline
\end{tabular}

${ }^{*}$ ) Al sexto grupo, denominado Control del Procedimiento Quirúrgico (SHAM), se le realizó toda la manipulación quirúrgica sin llegar a extirpar los ovarios. OVX: ovariectomía.

pequeñas cantidades de manera natural en el forraje y se le atribuyen también efectos antimicrobianos y fungicidas ${ }^{(10,11)}$.

Bajo ciertas condiciones, la cantidad de coumestrol en la alfalfa puede llegar a superar los 100 $\mathrm{mg} / \mathrm{kg}$ materia seca (MS); cantidad elevada que puede causar, en las hembras que consumen dicho forraje, aumento en la repetición de celos, abortos, metritis y quistes ováricos; patologías que se agrupan bajo el término de "síndrome estrogénico" (12).

Precisamente por su actividad estrogénica, se ha difundido su uso para aliviar los síntomas propios de la menopausia. La doble actividad de las isoflavonas (actuando a la vez como estrogénicas y antiestrogénicas), le confieren una serie de cualidades que permiten regular el balance hormonal en la mujer, pudiendo prevenir la osteoporosis y actuar como potentes antioxidantes que protegen frente al cáncer de mama. Asimismo, contribuyen a disminuir la intensidad y frecuencia de los sofocos, fatiga, sudor nocturno y cambios en el estado de ánimo ${ }^{(13,14)}$.

Uno de los modelos para evaluar actividad estrogénica es el incremento del peso del útero en hembras ovariectomizadas (OVX). Este efecto es fundamental y reproducible en animales expuestos a agonistas estrogénicos. El modelo de hembra adulta ovariectomizada consiste en la extirpación mediante cirugía de ambos ovarios. De esta manera se elimina la
Tabla 2. Análisis fitoquímico cualitativo del extracto hidroalcohólico de Medicago sativa L.

\begin{tabular}{lcc}
\hline \multicolumn{1}{c}{ Reactivo } & $\begin{array}{c}\text { Metabolito } \\
\text { secundario }\end{array}$ & Resultado \\
\hline Dragendorf & Alcaloides & ++ \\
Shinoda & Flavonoides & ++ \\
Tricloruro & $\begin{array}{c}\text { Compuestos } \\
\text { fenólicos }\end{array}$ & - \\
férrico & Taninos & ++ \\
Gelatina & Triterpenoides & - \\
Lieberman- & yesteroides & + \\
Burchard & Lactonas & + \\
Baljet A y B & Núcleo & + \\
Salkowski & esteroidal & - \\
Borntrager & Quinonas & + \\
Espuma & Saponinas & ++ \\
\hline
\end{tabular}
de síntesis de estrógenos $(15$, 16). Por lo tanto,

Abundante cantidad: +++ ; Regular cantidad: ++ ; Poca cantidad: + ; Ausencia: -

el incremento del peso relativo y absoluto de los cuernos uterinos en estos animales es el principal indicativo de que el extracto o droga empleada presenta actividad estrogénica ${ }^{(17)}$ y estaría causado por la interacción del estrógeno administrado con receptores que presentan actividad en los tejidos del útero, lo cual induce cambios

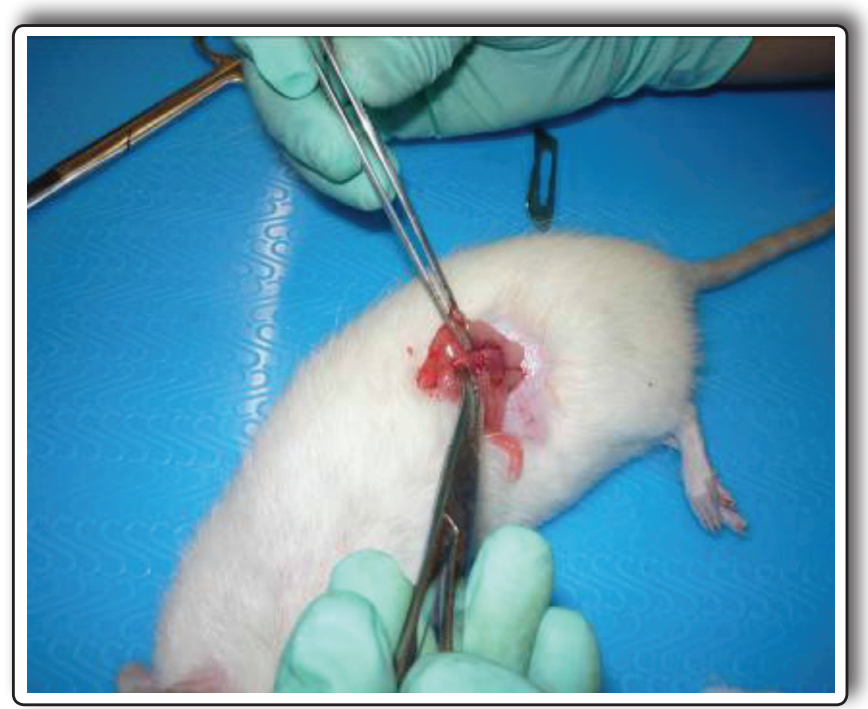

Figura 1. Abordaje bilateral por ambos flancos. Visualización del cuerno uterino izquierdo.

Tabla 3. Efecto del extracto hidroalcohólico de Medicago sativa alfalfa sobre el peso corporal en ratas OVX,

\begin{tabular}{|c|c|c|c|c|c|}
\hline \multirow{2}{*}{ Grupos } & \multirow{2}{*}{ Tratamiento } & \multirow{2}{*}{ Dosis } & \multicolumn{3}{|c|}{ Peso corporal (g) } \\
\hline & & & Peso 0 & Peso inicial & Peso final \\
\hline Control Negativo & Vehículo & $2 \mathrm{~mL} / \mathrm{kg}$ & $220,13 \pm 2,90$ & $307,13 \pm 6,8 *$ & $314,0 \pm 3,7^{*+}$ \\
\hline Control Positivo & Estradiol & $3 \mu \mathrm{g} / \mathrm{kg}$ & $220,75 \pm 3,37$ & $302,00 \pm 8,3 *$ & $296,25 \pm 6,71 *$ \\
\hline Experimento & Extracto alfalfa & $100 \mathrm{mg} / \mathrm{kg}$ & $219,75 \pm 2,82$ & $302,88 \pm 6,4 *$ & $308,75 \pm 8,29 *+$ \\
\hline Experimento & Extracto alfalfa & $500 \mathrm{mg} / \mathrm{kg}$ & $220,25 \pm 3,81$ & $301,25 \pm 6,7 *$ & $314,5 \pm 6,8^{*+}$ \\
\hline Experimento & Extracto alfalfa & $1000 \mathrm{mg} / \mathrm{kg}$ & $219,75 \pm 3,45$ & $302,13 \pm 5,4 *$ & $305,75 \pm 4,3 *+$ \\
\hline (CPQ) SHAM & Alimento/Agua & Ad libitum & $221,38 \pm 3,11$ & $267,75 \pm 6,6$ & $281,25 \pm 3,3$ \\
\hline
\end{tabular}

$\left.{ }^{*}\right) p<0,05$ Comparación con el grupo SHAM (ANOVA). Los resultados fueron expresados como Media \pm DE.

$(+) p<0,05$ Comparación con el grupo Control positivo (ANOVA). Los resultados fueron expresados como Media \pm DE. 
celulares que traen como consecuencia imbibición de agua en los tejidos y en el lumen uterino ${ }^{(18)}$.

El objetivo del presente trabajo fue determinar el efecto estrogénico del extracto hidroalcohólico de la especie Medicago sativa L (alfalfa) en el modelo de ratas albinas ovariectomizadas (OVX).

\section{MATERIALES Y MÉTODOS}

El estudio se realizó en la Facultad de Medicina de la Universidad Nacional Mayor de San Marcos. Se recolectó la planta entera (hojas, tallos y flores) sin raíz en la provincia de Canta, región Lima, en los meses de abril y mayo. Se identificó en el Herbario de la Universidad Nacional Agraria La Molina. La muestra fresca se llevó a una estufa con aire circulante a una temperatura de $40^{\circ} \mathrm{C}$ para su secado.

\section{Obtención del extracto hidroalcohólico de Medicago sativa $\mathrm{L}$ alfalfa}

Se emplearon 680 gramos de muestra seca y molida, los cuales fueron macerados en 7 litros de etanol a $96^{\circ}$, al que se añadió agua destilada en una proporción de 2:8 (agua:etanol). El tiempo de maceración fue por un periodo de 21 días bajo protección de la luz y calor ${ }^{(19)}$. Posteriormente se filtró la solución y se concentró en rotavapor, para luego ser colocada en estufa a una temperatura de $37^{\circ} \mathrm{C}$ hasta obtener una masa homogénea de consistencia blanda. Se realizó el análisis fitoquímico del extracto hidroalcohólico obtenido.

\section{Tamaño de muestra, distribución y tratamiento de los animales}

Se emplearon 48 ratas albinas Sprague Dawley de 8 semanas de edad, las cuales fueron alojadas en jaulas metálicas de crianza para su aclimatación, con libre acceso a agua y alimento, distribuyéndose en seis grupos constituidos por ocho ratas cada grupo, tal como se indica en la tabla 1. El extracto hidroalcohólico de Medicago sativa se administró durante 14 días por vía oral.

\section{Técnica de Ovariectomía (OVX)}

Se seleccionaron al azar 40 ratas, las cuales se mantuvieron en ayunas ocho horas antes de la intervención, anestesiándolas por vía intramuscular con una combinación de xilazina $(3 \mathrm{mg} / \mathrm{kg}$ ) y ketamina $(50 \mathrm{mg} / \mathrm{kg})$, luego se colocó a los animales en posición de decúbito lateral derecho, realizando una incisión de aproximadamente $1 \mathrm{~cm}$ en el cuadrante inferior del abdomen (fosa del ijar izquierdo), se alcanza el ovario que se separa con una ligadura junto con unos $5 \mathrm{~mm}$ del cuerno uterino correspondiente y se extrae de la cavidad peritoneal. La incisión se cierray se procede de igual forma al lado derecho. Entre los diez y quince días postcirugía se realizaron frotices vaginales de cada uno de los animales, para comprobar que las ratas ovariectomizadas se encontraran en fase diestro o fase inactiva (figura 1).

\section{Parámetros evaluados}

Peso corporal de los animales: en balanza analítica al inicio y al término del tratamiento.

Tabla 4. Efecto del extracto hidroalcohólico de Medicago sativa alfalfa sobre el peso del útero en ratas OVX.

\begin{tabular}{lcccc}
\hline \multicolumn{1}{c}{ Grupos } & Tratamiento & Dosis & \multicolumn{2}{c}{ Peso corporal (g) } \\
\cline { 3 - 5 } & & $2 \mathrm{~mL} / \mathrm{kg}$ & Absoluto (g) & Relativo (\%) \\
\hline Control Negativo & Vehículo & $3 \mu \mathrm{g} / \mathrm{kg}$ & $0,22 \pm 0,07$ & $0,07 \pm 0,03$ \\
Control Positivo & Estradiol & $100 \mathrm{mg} / \mathrm{kg}$ & $1,45 \pm 0,21^{*+}$ & $0,52 \pm 0,07 *+$ \\
Experimento & Extracto alfalfa & $500 \mathrm{mg} / \mathrm{kg}$ & $0,29 \pm 0,06$ & $0,10 \pm 0,02$ \\
Experimento & Extracto alfalfa & $1000 \mathrm{mg} / \mathrm{kg}$ & $0,38 \pm 0,03 *$ & $0,13 \pm 0,03 *$ \\
Experimento & Extracto alfalfa & Ad libitum & $0,66 \pm 0,04 * \bullet$ & $0,22 \pm 0,04^{* \bullet \bullet}$ \\
(CPQ) SHAM & Alimento/Agua & $0,79 \pm 0,08 *$ & $0,28 \pm 0,03 *$ \\
\hline
\end{tabular}

$\left(^{*}\right) p<0,05$ Comparación con el grupo control negativo (ANOVA). (+) $p<0,05$ Comparación con los demás grupos (ANOVA). (*) p<0,05 Comparación con el grupo 3 (ANOVA). Los resultados fueron expresados como Media \pm DE.

Tabla 5. Efecto del extracto hidroalcohólico de Medicago sativa alfalfa sobre la citología vaginal en ratas OVX.

\begin{tabular}{|c|c|c|c|c|c|c|}
\hline \multirow{2}{*}{ Grupos } & \multirow{2}{*}{ Tratamiento } & \multirow{2}{*}{ Dosis } & \multicolumn{4}{|c|}{ Citología vaginal } \\
\hline & & & $24 h$ & $48 h$ & $72 \mathrm{~h}$ & $96 \mathrm{~h}$ \\
\hline Control Negativo & Vehículo & $2 \mathrm{~mL} / \mathrm{kg}$ & Diestro & Diestro & Diestro & Diestro \\
\hline Control Positivo & Estradiol & $3 \mu \mathrm{g} / \mathrm{kg}$ & Proestro & Estro & Metaestro & Diestro \\
\hline Experimento & Extracto alfalfa & $100 \mathrm{mg} / \mathrm{kg}$ & Diestro & Diestro & Diestro & Diestro \\
\hline Experimento & Extracto alfalfa & $500 \mathrm{mg} / \mathrm{kg}$ & Diestro & Diestro & Diestro & Diestro \\
\hline Experimento & Extracto alfalfa & $1000 \mathrm{mg} / \mathrm{kg}$ & Diestro & Diestro & Diestro & Proestro \\
\hline (CPQ) SHAM & Alimento/Agua & Ad libitum & Proestro & Estro & Metaestro & Diestro \\
\hline
\end{tabular}




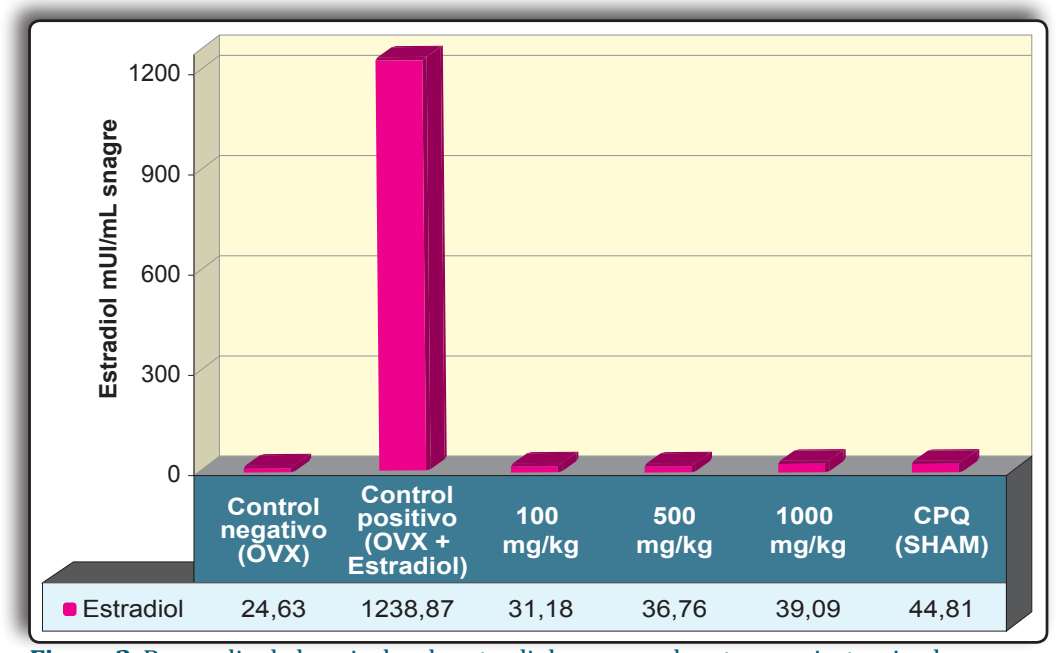

Figura 2. Promedio de los niveles de estradiol en suero de ratas ovariectomizadas.

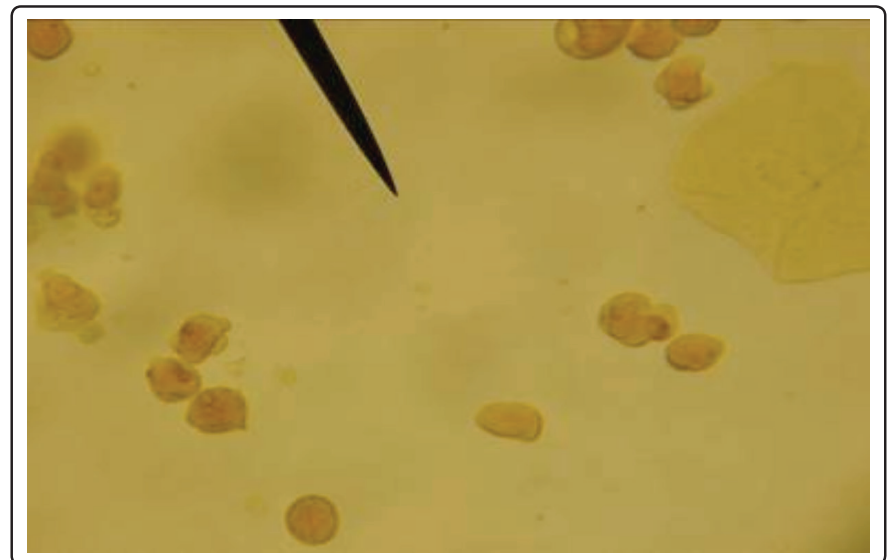

Figura 3. Citología vaginal de rata ovariectomizada, fase diestro. 100x.

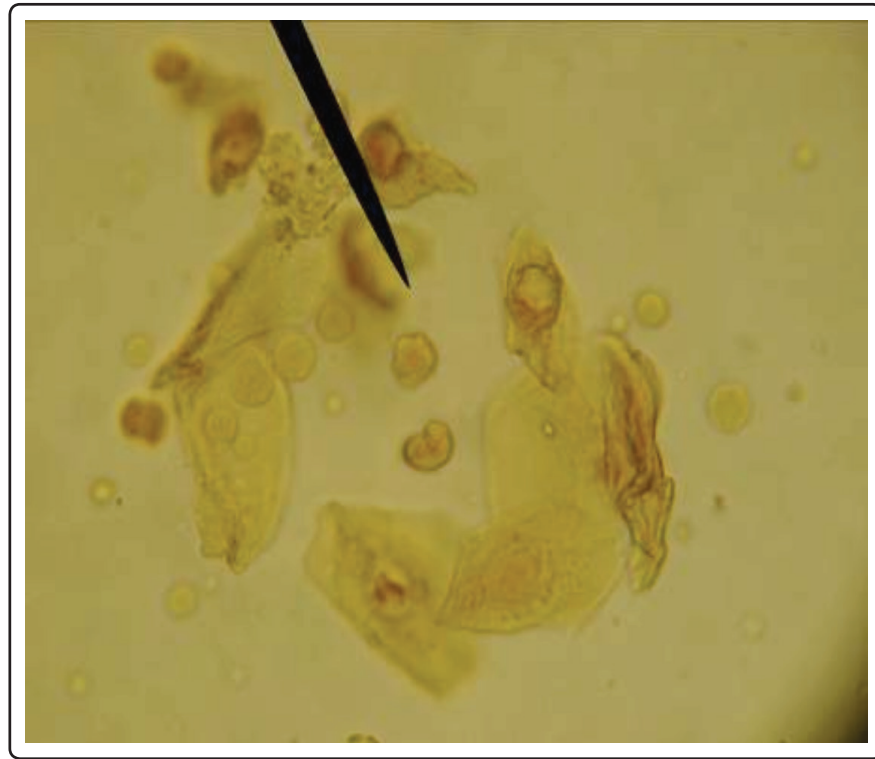

Figura 4. Citología vaginal de rata ovariectomizada, fase estro (células cornificadas). $100 x$.
Peso del útero: el que fue separado de la vagina por el cuello uterino e inmediatamente pesado en balanza analítica.

Cambios en el ciclo estral: mediante el estudio del frotis vaginal a las $24,48,72$ y 96 horas del inicio de la administración, vía oral, del extracto hidroalcohólico.

Análisis del perfil hormonal: mediante la técnica de quimioluminiscencia, se determinaron los niveles de estradiol en plasma sanguíneo de los animales.

\section{Análisis estadístico}

La diferencia entre los grupos tratados se determinó mediante análisis de varianza ANOVA. Para las comparaciones entre grupos se utilizó el Test Tukey, considerándose significativo $p<0,05$. Todo el procedimiento se realizó con el programa SPSS (Stadistical Package for Socials Sciences), versión 15.0 en español.

\section{RESULTADOS}

\section{De la técnica quirúrgica ovariectomía (OVX) en ratas albinas}

La combinación anestésica xilazina y Ketamina indujo un plano quirúrgico óptimo (plano 2) para realizar la ovariectomía bilateral por el flanco (OVX), sin causar complicaciones

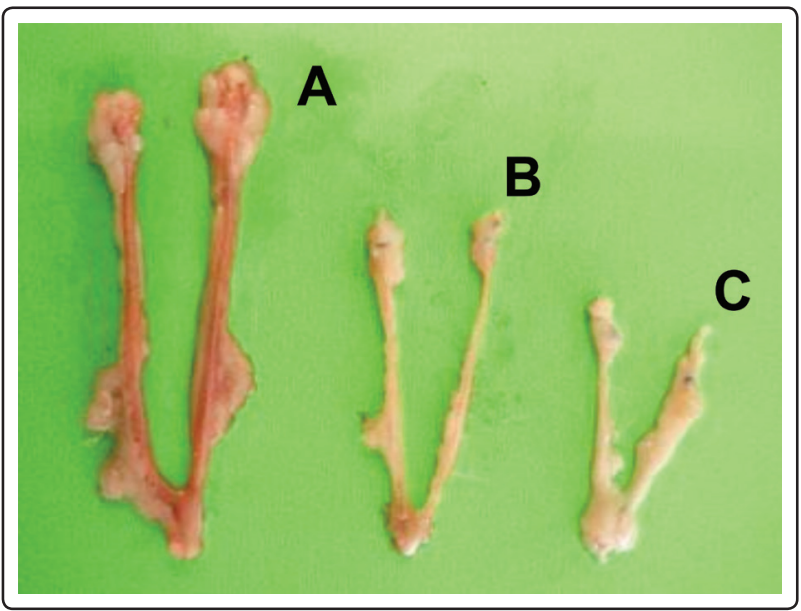

Figura 5. Involución de Cuernos Uterinos postcirugía. A: Rata no ovariectimizada (No OVX): cuernos uterinos y ovarios. B: Rata ovariectimizada, cuernos uterinos sin ovarios, 02 semanas postcirugía. C: Rata ovariectimizada, cuernos uterinos sin ovarios, 04 semanas postcirugía. 
en el postoperatorio, ni la muerte de ninguno de los animales. El tiempo de duración de la anestesia obtenida con este protocolo fue de 40 minutos aproximadamente y el tiempo de recuperación fue de 4-6 horas al término de los cuales los animales iniciaban la ingesta de alimento y agua.

\section{De los parámetros evaluados}

Los resultados referentes al peso corporal, peso del útero, citología vaginal y niveles de estradíol, pueden observarse en las tablas 3 a 5 y figuras 2 a 5 , respectivamente.

\section{DISCUSIÓN}

De las veinticuatro variedades de alfalfa que son cultivadas en la sierra de nuestro país ${ }^{(21)}$, para el presente estudio sólo se ha considerado la variedad "Moapa Superior", recolectada en la provincia de Canta, yaunque todavía no se ha determinadola concentración de coumestrol en dicha variedad, ésta puede verse afectada por los cambios de temperaturas, fotoperíodo y régimen hídrico en que se cultivan ${ }^{(21)}$; por lo que se deben tener en cuenta al formular suplementos estrogénicos para uso humano provenientes de esta leguminosa.

La acción que tiene la vitamina $\mathrm{K}$ sobre la osteocalcina podría trabajar en forma sinérgica con el coumestrol, para la prevención de osteoporosis en mujeres menopáusicas; ambos se encuentran en la alfalfa ${ }^{(22)}$.

El contenido de alcaloides hallado fue en regular cantidad, lo que podría atribuirse al estado de floración de la variedad de alfalfa "Moapa superior", el cual llega a un $20 \%$ de floración lo cual es indicativo del estado de madurez de la planta ${ }^{(23)}$. Asimismo, se ha determinado la presencia de saponinas en regular cantidad, la que no está relacionada al contenido de coumestrol en esta leguminosa, aunque hasta la fecha, no se ha publicado ninguna investigación relacionando ambos compuestos ${ }^{(24)}$.

En relación a la técnica de ovariectomía bilateral realizada en las ratas albinas (figura 2), el protocolo de anestesia empleado utilizando xilazina $(3 \mathrm{mg} / \mathrm{kg}$ ) y ketamina $(50 \mathrm{mg} / \mathrm{kg}$ ), proporcionó un buen plano anestésico con un óptimo grado de analgesia sumado a una pronta recuperación de los animales ${ }^{(16)}$.

Los hallazgos estadísticos indican que en las condicionesexperimentales, elextractohidroalcohólico de Medicago sativa aumentó los valores sanguíneos de estradiol en ratas albinas ovariectomizadas en comparación con el grupo control negativo, sin embargo, estos valores no fueron suficientes para provocar cambios en la citología vaginal (figuras 3 y 4), con excepción del grupo 5 que recibió una dosis de $1000 \mathrm{mg} / \mathrm{kg}$ (proestro al cuarto día de iniciado la administración del extracto); pero si fueron suficientes para provocar un marcado incremento en el peso del útero con respecto al control negativo (figura 5). Los niveles hormonales de estradiol fueron directamente proporcionales a la concentración de los extractos hidroalcohólicos administrados. Los resultados relacionados con los pesos del útero, absolutoy relativo, así como del análisis serológico en los grupos control negativo, CPQ (SHAM) y control positivo demuestran la consistencia del modelo de ratas ovariectomizadas (OVX) empleado con el tratamiento del extracto hidroalacohólico de Medicago sativa alfalfa. Por lo que se recomienda realizar posteriores estudios sobre efecto estrogénico de otras variedades de Medicago sativa separando el contenido de clorofila y demás compuestos que pudieran interferir con la actividad y cantidad de los fitoestrógenos contenidos.

\section{CONCLUSIONES}

- El extracto hidroalcohólico de Medicago sativa alfalfa presentó efecto estrogénico al aumentar el peso del útero y los niveles de estradiol en las ratas ovariectomizadas.

- Los metabolitos secundarios: flavonoides, saponinas, taninos y alcaloides se encuentran en regular cantidad.

\section{REFERENCIAS BIBLIOGRÁFICAS}

1. Rossouw J, Prentice R, Manson J, Wu L, Barad D, Barnabei $\mathrm{V}$, Ko $\mathrm{M}$ et al. Postmenopausal hormone therapy and risk of cardiovascular disease by age and years since menopause. JAMA 2007; 297 (13): 1465-77.

2. Matthews K, Kuller L, Sutton-Tyrrell K, Chang Y. Changes in cardiovascular risk factors during the perimenopause and postmenopause and carotid artery atherosclerosis in healthy women. Stroke. 2001; 32(5): 1104-11.

3. McEwen B, Stephen A. Estrogen actions in the central nervous system. Endocrine Reviews 1999; 20(3): 279-307.

4. Leiblum S, Bachmann G, Kemmann E, Colburn D, Swartzman L. Vaginal atrophy in postmenopausal women. The importance of sexual activity and hormones. JAMA 1983; 249(16): 2195-8.

5. Geller S, Studee L. Botanical and dietary supplements for menopausal symptoms: what works, what doesn't. J Womens Health (Larchmt) 2005; 14(7): 634-49. 
6. Geller S, Studee L. Soy and red clover for mid-life and aging. Climacteric 2006; 9(4): 245-63.

7. Adams NR. Detection of the effects of phytoestrogens on sheep and cattle. J Anim Sci 1995; 73(5): 1509-15.

8. Ravnikar VA. Compliance with hormone therapy. Am J Obstet Gynecol 1987; 156(5): 1332-36

9. Boué SM, Wiese TE, Nehls S, Burow ME, Elliott S, CarterWientjes $\mathrm{CH}$, et al. Evaluation of the estrogenic effects of legume extracts containing phytoestrogens. J Agric Food Chem 2003; 51(8): 2193-9.

10. Bickoff AM, Livingston AL. Forage estrogens, relative potencies of several estrogenlike compounds found in forages. J Agric Food Chem. 1962; 10(5): 410-2.

11. Cowan MM. Plants products as antimicrobial agents. Clin. Microbiol Rev. 1999; 12(4): 564-82.

12. Le Bars J, Le Bars P, Et Brice G. Presence accumulation et devenir du coumestrol dans la luzerne et ses derives. Rec Med Vet 1990; 166(5): 463-9.

13. Burton JJ, Wells M. The effect of phytoestrogens on the female genital tract. J Clin Pathol 2002; 55(6): 401-7.

14. Nikander E, Metsä-Heikkilä M, Ylikorkala O, Tiitinen A. Effects of phytoestrogens on bone turnover in postmenopausal women with a history of breast cancer. J Clin Endocrinol Metab 2004; 89(3): 1207-12.

15. Mei J, Yeung SS, Kung AW. High dietary phytoestrogen intake is associated with higher bone mineral density in postmenopausal but not premenopausal women. J Clin Endocrinol Metab 2001; 86(11): 5217-21.

16. Davidge S, Zhang Y, Stewart KG. A comparison of ovariectomy models for estrogen studies. Am J Physiol Regul Integr Comp Physiol 2001; 280(3): R904-7.

17. Kanno J, Onyon L, Haseman J, Fenner-Crisp P, Ashby J, Owens W. The OECD program to validate the rat uterotrophic bioassay to screen compounds for in vivo estrogenic responses: phase 1. Environ Health Perspect 2001; 109(8): 785-94.
18. Kanno J, Onyon L, Peddada S, Ashby J, Jacob E, Owens $\mathrm{W}$. The OECD program to validate the rat uterotrophic bioassay. Phase 2: coded single-dose studies. Environ Health Perspect. 2003; 111(12): 1550-8.

19. CYTED. Manual de Técnicas de Investigación. Programa Iberoamericano de Ciencia y Tecnología para el Desarrollo. Proyecto X-1. Búsqueda de principios bioactivos en plantas de la región. 1995. p. 220.

20. Lock De Ugaz O. Investigación Fitoquímica. $2^{\text {da }}$ ed. Pontificia Universidad Católica del Perú. Lima, 1994.

21. Noli C. Producción de forrajes para cuyes. INIA. Curso Nacional de crianza de cuyes. Marzo 15-16 de 2005. Huancayo - Perú, 2005.

22. Cheung A, Tile L, Lee Y, TomLinson G, Hawker G, Scher J, Hu $\mathrm{H}$, et al. Vitamin $\mathrm{K}$ supplementation in postmenopausal women with osteopenia (ECKO Trial): A Randomized Controlled Trial. PLoS Med 2008; 5(10): 1461-72.

23. Stochmal A, Piacente S, Pizza C, De Riccardis F, Leitz R, Oleszek W. Alfalfa (Medicago sativa L.) flavonoids. 1. Apigenin and luteolin glycosides from aerial parts. J Agric Food Chem 2001; 49(2): 753-58.

24. Anaya AL, Espinosa-García F, Cruz-Ortega R, AbouMansour E. Relaciones químicas entre organismos: Aspectos básicos y perspectivas de su aplicación. Plaza y Váldez. México, 2001.

Manuscrito recibido el: o6/10/14

Aceptado para su publicación el: 20/10/14

\section{Correspondencia:}

Nombre: $\quad$ Sandra G. Bezada

Dirección: Jr. Puno 1002 - Lima o1

Email: $\quad$ marcofi71@yahoo.es 